\title{
Relationship between IL-23R rs11209026 Gene Polymorphism and Susceptibility to Acute Coronary Syndrome
}

\author{
Dong Wang1*, Qian $\mathrm{Li}^{2 *}$, Shibao Song1, Chengbin $\mathrm{Li}^{2 \#}$ \\ ${ }^{1}$ The Second Clinical Medical College, Yangtze University, Jingzhou, China \\ ${ }^{2}$ Department of Laboratory Medicine, Jingzhou Central Hospital, The Second Clinical Medical College, Yangtze University, \\ Jingzhou, China \\ Email: "jzlcb002@163.com
}

How to cite this paper: Wang, D., Li, Q., Song, S.B. and Li, C.B. (2018) Relationship between IL-23R rs11209026 Gene Polymorphism and Susceptibility to Acute Coronary Syndrome. Yangtze Medicine, 2, 192-202.

https://doi.org/10.4236/ym.2018.23021

Received: July 27, 2018

Accepted: August 27, 2018

Published: August 30, 2018

Copyright $\odot 2018$ by authors and Scientific Research Publishing Inc. This work is licensed under the Creative Commons Attribution International License (CC BY 4.0).

http://creativecommons.org/licenses/by/4.0/

(c) (i) Open Access

\begin{abstract}
Background: Several studies illustrated that IL-23/Th17 axis could be an important pro-inflammatory pathway in atherosclerosis. As a key element in this inflammatory mechanism, interleukin-23 receptor (IL-23R) may play a critical role in the pathological process of atherosclerosis. Single nucleotide polymorphisms (SNPs) in IL-23R have recently been consistently found to be associated with atherosclerosis diseases. However, its association with acute coronary syndrome (ACS) is still indistinct. Here, we discussed whether genetic polymorphisms in IL-23R (rs11209026 G/A) were associated with susceptibility to ACS. Methods: Among 160 patients with ACS, it includes 80 patients with unstable angina pectoris (UAP), 80 patients with myocardial infarction (MI), and 80 control subjects were selected randomly. The polymorphisms of IL-23R ( $r 11209026$ G/A) were analyzed by the Sanger method. Results: Data showed that percentages of rs11209026 AG genotypes were significantly lower in ACS group (including: UAP and MI) than in controls (odds ratio $[\mathrm{OR}]=0.324,95 \%$ confidence interval $[\mathrm{CI}]: 0.148-0.712, \mathrm{p}=$ 0.005; OR $=0.351,95 \%$ CI: $0.135-0.910, p=0.031$; OR $=0.303,95 \%$ CI: $0.112-0.817, \mathrm{p}=0.018$, respectively). Furthermore, there was no correlation between rs11209026 G/A SNP and dyslipidemia-associated ACS. Conclusions: The variant of the rs11209026 polymorphism in IL-23R gene might decrease the risk of ACS, and these data suggest that AG genotype of the rs 11209026 G/A polymorphism may act as a protective factor for acute coronary syndrome and subtype of ACS.
\end{abstract}




\section{Keywords}

IL-23R, Gene Polymorphism, Acute Coronary Syndrome

\section{Introduction}

As a major public health issue, coronary heart disease (a clinical form of coronary atherosclerosis) has been the leading cause of mortality and morbidity worldwide [1]. More and more evidence has confirmed that atherosclerosis is a chronic inflammatory disease of the arterial vessel wall. Many inflammatory cells and inflammatory mediators play an important role in the occurrence, development and thrombosis among atherosclerotic lesions [2]. Acute coronary syndrome (ACS) is a clinical syndrome of coronary heart disease (CHD); it is a progressive inflammatory disease of the coronary arterial vascular wall and remains among high risk of mortality, which includes unstable angina pectoris (UAP), non-ST elevation myocardial infarction (NSTEMI), and ST elevation myocardial infarction (STEMI) [1] [2].

The key mechanism of ACS is atherosclerotic plaque rupture or erosion and platelet thrombus could occlude the lumen of the atherosclerotic coronary. Atherosclerotic plaque rupture is the key mechanism of ACS. Inflammation plays an important role in the rupture of the vulnerable plaques by regulating the stability of atherosclerotic plaque, as well as the plaque's thrombogenic potential [3]. To understand the mechanisms of inflammation in AS and ACS, several systemic inflammatory markers have been indicated as risk factors in cardiovascular events, especially in ACS [4]. Recently, a new potential marker IL-23 has drawn great attention. Interleukin (IL)-23 is a heterodimeric proinflammatory cytokine composed of a 19 subunit and a p 40 subunit, which is also part of IL-12 [5]. IL-23 is mainly produced by activated DCs, monocytes, and macrophages. The biological effect of IL-23 is achieved by binding to the specific with high affinity IL-23 receptor (IL-23R) on the surface of the target cell [6]. The IL-23R heterodimeric complex is composed of the IL-12R $\beta 1$ subunit and another specific IL-23R subunit, which is considered to be essential for the Th17 cell mediated immune response. Type 17 helper cells (Th17) are a recently discovered lineage of proinflammatory $\mathrm{CD} 4^{+} \mathrm{T}$ cells and IL-17 is a main effect cytokine produced by Th17 [7].

Moreover, abundant attention has focused on the novel inflammation pathway: IL-23/Th17 axis, which has been shown to play a central role in chronic inflammatory and autoimmune diseases. Notably, previous researches tended to consider IL-23R, and IL-23 plays a crucial role in initiating, maintaining and accelerating the IL-23/Th17 inflammatory signal transduction pathway. Several studies indicate that IL-23 can be involved in the development of atherosclerosis. Researchers have shown that mRNA levels of IL-23 and IL-23R were markedly 
increased in carotid plaques and IL-23 increased IL-17 release in monocytes and particularly in peripheral blood mononuclear cells that could aggravate carotid atherosclerosis, which indicated the possible role for the IL-23/Th17 Axis [8]. Another report has presented that IL-23R rs6682925T/C polymorphism may act as a risk factor of $\mathrm{CHD}$ and subjects carrying rs6682925TC and CC genotypes had significantly increased mRNA level of IL-23R in PBMCs than those with TT genotype [9]. Meanwhile, Kave et al. suggested that IL-23R rs11209026 G > A polymorphism is negatively correlated with the risk of AS. The polymorphism of this gene polymorphism may inhibit the progression of AS [10]. Furthermore, IL-23R was suggested to play an important part of the IL-23/Th17 pathway and it may also play critical roles in atherosclerosis and atherosclerosis-related diseases.

While various clinically relevant single-nucleotide polymorphisms (SNPs) sites have been reported in the IL-23R gene in order to explore the probability of genetic susceptibility to atherosclerosis. However, the subjects of case group in the previous studies were coronary heart disease and participants involved in the control group were healthy people (no coronary angiography). Furthermore, all subjects in present study were underwent coronary angiography, and the patients in case group were all patients with clinically diagnosed acute coronary syndrome. In addition, there are few studies explore the correlation between IL-23R gene polymorphisms and genetic susceptibility to acute coronary syndromes. In the current study, the subjects of control group were all with normal coronary artery, and patients in this case group were all acute coronary syndromes. Additionally, no study has explored the association of genetic susceptibility between rs $11209026 \mathrm{G} / \mathrm{A}$ and ACS. Therefore, our study aims to discuss the possible association between IL-23R rs11209026 G/A variants and incidence of ACS.

\section{Materials and Methods}

\subsection{Patients and Controls}

This case-control study recruited 240 patients who presented with cardiovascular symptoms to Jingzhou Central Hospital. All patients who underwent coronary angiography and other types of heart diseases, severe liver, kidney, brain or lung dysfunction, malignant tumor, autoimmune diseases, connective tissue diseases, infectious diseases were excluded from the study. Coronary stenosis was considered significant in the presence of a luminal diameter narrowing of $>50 \%$ of at least one pericardial coronary artery. According to the Foundation of the American College of Cardiology/American Heart Association (ACCF/AHA), the 240 patients were divided into case group (Acute coronary syndrome) and control group (normal coronary arteries). The cases group including $160 \mathrm{pa}$ tients and was divided into two subgroups. Unstable angina group including 80 patients with unstable angina pectoris (UAP), Myocardial infarction (MI) group contain 44 patients with non-ST-elevation myocardial infarction (NSTEMI) and 
36 patients with ST-elevation myocardial infarction (STEMI). The control group involved 80 patients who were normal coronary arteries. All the participants involved in this study completed a standard questionnaire about their personal history, which included data on age, sex, smoking status, history of hypertension, history of diabetes and some biochemical indicators obtained from patient cases. All individuals enrolled were from the Han population in China. This study was approved by the ethics committee of Jingzhou Center Hospital. All subjects were signed the informed consent.

\subsection{DNA Extraction and Genotyping}

Whole blood samples were collected in EDTA-K2 tubes from all subjects and then frozen. Genomic DNA was extracted from frozen whole blood using the Sangon Biotech DNA Extraction Kit and Purification Kits were used to purify genomic DNA of all subjects, and the DNA extracts were stored at $-70^{\circ} \mathrm{C}$ until the time of analysis. The rs $11209026 \mathrm{G} / \mathrm{A}$ polymorphisms were genotyped by the Sanger DNA sequencing method assay. The target sequencing fragment was amplified by the ABI 7500 PCR instrument (Applied Biosystems, Inc). Oligonucleotide primers were designed by Primer Premier 5 for the PCR. The applied primer sequences included 5'-AAACAGTCACTCTGTGGCCTAAAG-3' (Forward-Primer) and 5'-GGAAAGACATTTGT AGAGAGTTTGG-3' (Reverse-Primer). The total volume of the PCR was $50 \mu$, including $25 \mu \mathrm{l} 2 \times$ Taq PCR MasterMix, $2 \mu \mathrm{l}$ each primer $(10 \mu \mathrm{mol} / \mathrm{L}), 15 \mu \mathrm{l}$ Sterilized $\mathrm{ddH}_{2} \mathrm{O}, 6 \mu \mathrm{l}$ DNA template $(<1$ $\mu \mathrm{g})$. The reaction conditions of PCR: initial denaturation for 3 minutes at $95^{\circ} \mathrm{C}$, followed by 30 cycles of 30 seconds at $95^{\circ} \mathrm{C}$ (denaturation), 30 seconds at $54^{\circ} \mathrm{C}$ (annealing), 1 minute at $72^{\circ} \mathrm{C}$ (elongation), and 10 minutes at $72^{\circ} \mathrm{C}$ after 30 cycles (final elongation). $2 \%$ agarose electrophoresis was used to detect the specificity of the amplified products after the PCR reaction. The amplified products was a fragment of 443 base pairs The DNA sequencing of all amplified products was completed by Sangon Biotech (Wuhan) Co., Ltd. (Forward primer sequencing).

\subsection{Statistical Analysis}

Continuous data that was distributed normally was described using mean \pm standard deviations (SD); Continuous data that was distributed non-normally was described using median and quartile range; Categorical data was described using the number of cases and the percentage (\%). According to types of the data above, the Student t-test/one-way anova, the Mann-Whitney test and the chi-square $\left(\chi^{2}\right)$ test were used to analyse the difference of groups respectively. The $\chi^{2}$ goodness-of-fit test was used to analyse Hardy-Weinberg equilibrium. The crude odds ratios (OR) with $95 \%$ confidence interval (CI) were used to assess the frequencies of genotypes and alleles. A two-tailed p-value of $<0.05$ has been considered statistically significant. Statistical Package for Social Science (SPSS) software version 23 was used for the general statistical analyses. 


\section{Results}

\subsection{Clinical Characteristics of Patients and Controls}

The clinical characteristics of all the participants are shown in Table 1. There was no significant difference in gender $(p>0.05)$, age $(p>0.05)$, TC (Total Cholesterol) ( $\mathrm{p}>0.05)$, LDL-C (Low Density Lipoprotein Cholesterol) $(\mathrm{p}>$ 0.05), Apolipoprotein B ( $p>0.05)$ between the ACS patients and the Control group subjects (normal coronary arteries). And the participants of ACS comparison with the controls, UAP and MI patients exhibited higher level of FBG, TG, HDL-C and BMI, increased frequency of smoking, hypertension, diabetes and dyslipidemia, all of which are the defined risk factors of ACS (Table 1).

\subsection{Distributions of the IL-23R rs11209026 Polymorphisms in the ACS Cases and Controls}

Detection of IL-23R rs11209026 polymorphisms genotypes by Sanger method and the genotype distributions of SNP of IL-23 receptor and allele frequencies are summarized in Table 2. Genotypes of the rs11209026 polymorphism among

Table 1. Clinical characteristics of participants in the study.

\begin{tabular}{|c|c|c|c|c|c|c|}
\hline \multirow{2}{*}{ Characteristics } & \multicolumn{2}{|c|}{ Subtype of ACS } & \multirow{2}{*}{$\begin{array}{l}\text { Controls } \\
(\mathrm{n}=80)\end{array}$} & \multicolumn{3}{|c|}{$\mathrm{P}$} \\
\hline & $\begin{array}{c}\text { UAP } \\
(\mathrm{n}=80)\end{array}$ & $\begin{array}{c}\text { MI } \\
(\mathrm{n}=80)\end{array}$ & & $\begin{array}{l}\text { MI vs } \\
\text { controls }\end{array}$ & $\begin{array}{l}\text { UAP vs } \\
\text { controls }\end{array}$ & $\begin{array}{c}\text { MI } \\
\text { vs UAP }\end{array}$ \\
\hline Gender (M/F) & $50 / 30$ & $47 / 33$ & $43 / 37$ & $>0.05$ & $>0.05$ & $>0.05$ \\
\hline Age (years, mean \pm SD) & $60.66 \pm 9.05$ & $59.23 \pm 10.04$ & $58.39 \pm 9.43$ & $>0.05$ & $>0.05$ & $>0.05$ \\
\hline BMI $\left(\mathrm{kg} / \mathrm{m}^{2}\right)$ & $24.94 \pm 2.27$ & $25.9 \pm 4.24$ & $23.83 \pm 3.31$ & $>0.05$ & $>0.05$ & $>0.05$ \\
\hline Smoking n (\%) & $39(48.8)$ & $44(55)$ & $25(31.3)$ & $<0.01$ & $<0.05$ & $>0.05$ \\
\hline Hypertension n (\%) & $58(72.5)$ & $65(81.3)$ & $44(55)$ & $<0.01$ & $<0.01$ & $>0.05$ \\
\hline Diabetes n (\%) & $22(27.5)$ & $31(38.8)$ & $7(8.7)$ & $<0.01$ & $<0.01$ & $>0.05$ \\
\hline Dyslipidemia (\%) & $43(53.8)$ & $53(66.3)$ & $18(25.7)$ & $<0.01$ & $<0.01$ & $>0.05$ \\
\hline $\mathrm{FBG}(\mathrm{mmol} / \mathrm{L})$ & $6.28 \pm 2.58$ & $6.50 \pm 2.94$ & $5.12 \pm 0.71$ & $<0.01$ & $<0.01$ & $>0.05$ \\
\hline $\mathrm{TC}(\mathrm{mmol} / \mathrm{L})$ & $4.33 \pm 1.18$ & $4.39 \pm 1.14$ & $4.47 \pm 0.95$ & $>0.05$ & $>0.05$ & $>0.05$ \\
\hline TG (mmol/L) & $2.23 \pm 1.95$ & $1.74 \pm 0.99$ & $1.33 \pm 0.26$ & $<0.01$ & $<0.01$ & $>0.05$ \\
\hline LDL-C (mmol/L) & $2.28 \pm 0.89$ & $2.55 \pm 1.04$ & $2.53 \pm 0.61$ & $>0.05$ & $>0.05$ & $>0.05$ \\
\hline HDL-C $(\mathrm{mmol} / \mathrm{L})$ & $1.13 \pm 0.29$ & $1.08 \pm 0.31$ & $1.33 \pm 0.26$ & $<0.01$ & $<0.01$ & $>0.05$ \\
\hline Apob (g/L) & $0.85 \pm 0.29$ & $0.93 \pm 0.27$ & $0.86 \pm 0.20$ & $>0.05$ & $>0.05$ & $>0.05$ \\
\hline $\mathrm{UA}(\mu \mathrm{mol} / \mathrm{L})$ & $383.29 \pm 99.79$ & $364.44 \pm 91.57$ & $320.96 \pm 85.93$ & $<0.01$ & $<0.01$ & $>0.05$ \\
\hline $\mathrm{BUN}(\mathrm{mmol} / \mathrm{L})$ & $5.53 \pm 1.93$ & $5.45 \pm 1.71$ & $4.85 \pm 1.38$ & $<0.05$ & $<0.05$ & $>0.05$ \\
\hline $\mathrm{Cr}(\mu \mathrm{mol} / \mathrm{L})$ & $73.90 \pm 23.49$ & $81.68 \pm 22.45$ & $64.18 \pm 21.05$ & $<0.01$ & $<0.01$ & $<0.05$ \\
\hline
\end{tabular}

UAP: unstable angina pectoris; MI: myocardial infarction; BMI: body mass index; FBG: Fasting Blood Glucose; TC: Total Cholesterol; TG: Triglyceride; LDL-C: Low Density Lipoprotein Cholesterol; HDL-C: High Density Lipoprotein Cholesterol; Apob: Apolipoprotein B; UA: Uric acid; BUN: blood urea nitrogen; Cr: creatinine; $\mathrm{P}<0.05$ is significant. 
Table 2. The genotypes and alleles frequencies of the rs11209026 polymorphism within IL-23R gene in all groups.

\begin{tabular}{|c|c|c|c|c|c|c|c|c|c|c|}
\hline \multirow{3}{*}{ rs11209026 } & \multirow{3}{*}{$\begin{array}{c}\begin{array}{c}\text { Controls } \\
(\mathrm{n}=80)\end{array} \\
\mathrm{n}(\%)\end{array}$} & \multirow{2}{*}{\multicolumn{3}{|c|}{$\operatorname{ACS}(n=160)$}} & \multicolumn{6}{|c|}{ Subtype of ACS } \\
\hline & & & & & \multicolumn{3}{|c|}{$\mathrm{UAP}(\mathrm{n}=80)$} & \multicolumn{3}{|c|}{$\mathrm{MI}(\mathrm{n}=80)$} \\
\hline & & n (\%) & $x^{2}$ & $\mathrm{P}$ & n (\%) & $x^{2}$ & $\mathrm{P}$ & $\mathrm{n}(\%)$ & $x^{2}$ & $\mathrm{P}$ \\
\hline \multicolumn{11}{|l|}{ Genotypes } \\
\hline GG & $63(78.8)$ & 147 (91.9) & \multirow{2}{*}{8.400} & \multirow{2}{*}{0.004} & $73(91.3)$ & \multirow{2}{*}{4.902} & \multirow{2}{*}{0.027} & $74(92.5)$ & \multirow{2}{*}{6.144} & \multirow{2}{*}{0.013} \\
\hline AG & $17(21.3)$ & $13(8.1)$ & & & $7(8.8)$ & & & $6(7.5)$ & & \\
\hline \multicolumn{11}{|l|}{ Alleles } \\
\hline G & $143(89.4)^{2}$ & $307(95.9)^{a}$ & \multirow{2}{*}{7.840} & \multirow{2}{*}{0.005} & $153(95.5)^{\mathrm{a}}$ & \multirow{2}{*}{4.505} & \multirow{2}{*}{0.034} & $154(96.3)^{\mathrm{a}}$ & \multirow{2}{*}{5.668} & \multirow{2}{*}{0.017} \\
\hline A & $17(10.6)^{a}$ & $13(4.1)^{\mathrm{a}}$ & & & $7(4.4)^{\mathrm{a}}$ & & & $6(3.8)^{\mathrm{a}}$ & & \\
\hline
\end{tabular}

${ }^{\mathrm{a}}$ Frequency of a group of A alleles = total number of A alleles in the group/(total number of A alleles + total number of $\mathrm{G}$ alleles in the group).

the controls were in accordance with the Hardy-Weinberg equilibrium ( $\mathrm{p}>$ 0.05). As for the ACS cases and controls, percentages of GG and AG genotypes were $91.9 \%$ and $8.1 \%$ in the ACS patients $\left(\chi^{2}=8.400, \mathrm{P}=0.0004\right)$, and were $78.8 \%$ and $21.3 \%$ in the controls (normal coronary arteries). As for the subtype of ACS cases and the controls, frequency of GG and AG genotypes were 91.3\%, $8.8 \%$ in the UAP patients $\left(\chi^{2}=4.902, \mathrm{P}=0.027\right)$; were $92.5 \%, 7.5 \%$ in the MI patients $\left(\chi^{2}=6.144, \mathrm{P}=0.013\right)$, and were $78.8 \%$ and $21.3 \%$ in the controls. In addition, the AA genotype was not identified in the present study. Also, A allele of the $\mathrm{r}$ rs11209026G/A SNP showed clearly decreased percentage in ACS compared with controls $(4.1 \%$ vs. $10.6 \%, \mathrm{p}<0.005)$. Additionally, significant difference in the IL-23R A allele frequencies was detected in either UAP or MI patients contrasted with controls $(4.4 \%$ vs. $10.6 \%, \mathrm{p}<0.034 ; 3.8 \%$ vs. $10.6 \%, \mathrm{p}<$ $0.017)$.

\subsection{Associations of the Polymorphisms of IL-23R Gene (rs11209026) with ACS Risk}

Multinomial logistic regression results in current research adjusted by: age and sex. The Association analysis between genotype of rs11209026 and risk of ACS in case group and control group are reported in Table 3. From the results obtained so far, it seem that the risk of ACS patients who carriers with rs11209026 AG genotype was 0.324 times than those with AA genotype carriers (OR: 0.324, 95\% CI: 0.148 - 0.712, $\mathrm{p}=0.005)$. Similar tendencies were observed in UAP and MI group, patients with AG genotype 0.351 (UAP) and 0.303 (MI) times more odds of developing atherosclerosis than those with AG genotype (OR: 0.351, 95\% CI: $0.135-0.910, \mathrm{p}=0.031$ and OR: 0.303, 95\% CI: $0.112-0.817, \mathrm{p}=0.018$, respectively). These results indicate that $\mathrm{rs} 11209026 \mathrm{G} / \mathrm{A} \mathrm{SNP}$ is associated with decreased susceptibility to ACS.

\subsection{Association between rs11209026 G/A and Dyslipidemia in ACS}

Dyslipidemia is a crucial risk factor of ACS. We, therefore, explored whether 
rs11209026 G/A was associated with dyslipidemia in ACS. In the ACS group, 96 participants were diagnosed with dyslipidemia. Percentages of rs11209026 AG genotype and A allele were not significantly difference in patients with dyslipidemia than those without dyslipidemia $(\mathrm{OR}=1.067,95 \% \mathrm{CI}: 0.330-3.451, \mathrm{p}=$ 0.913 and $\mathrm{OR}=1.064,95 \% \mathrm{CI}: 0.337-3.356, \mathrm{p}=0.915$, respectively, Table 4). In order to survey whether rs 11209026 G/A SNP is independently correlated with dyslipidemia, we investigated this polymorphism with dyslipidemia in the normal coronary arteries group (control). However, there was no difference in the

Table 3. Association analysis between genotype of rs11209026 and risk of ACS in case group and control group by the logistic regression.

\begin{tabular}{|c|c|c|c|c|c|c|}
\hline \multirow{3}{*}{ rs11209026 } & \multirow{2}{*}{\multicolumn{2}{|c|}{$\operatorname{ACS}(n=160)$}} & \multicolumn{4}{|c|}{ Subtype of ACS } \\
\hline & & & \multicolumn{2}{|l|}{$\mathrm{UAP}(\mathrm{n}=80)$} & \multicolumn{2}{|l|}{$\mathrm{MI}(\mathrm{n}=80)$} \\
\hline & OR $(95 \% C I)^{\mathrm{a}}$ & $\mathrm{P}^{\mathrm{a}}$ & OR $(95 \% C I)^{\mathrm{a}}$ & $\mathrm{P}^{\mathrm{a}}$ & OR $(95 \% C I)^{\mathrm{a}}$ & $\mathrm{P}^{\mathrm{a}}$ \\
\hline \multicolumn{7}{|l|}{ Genotypes } \\
\hline GG & 1 & & 1 & & 1 & \\
\hline AG & $0.324(0.148-0.712)$ & 0.005 & $0.351(0.135-0.910)$ & 0.031 & $0.303(0.112-0.817)$ & 0.018 \\
\hline \multicolumn{7}{|l|}{ Alleles } \\
\hline G & 1 & & 1 & & 1 & \\
\hline A & $0.353(0.166-0.751)$ & 0.007 & $0.381(0.152-0.955)$ & 0.040 & $0.327(0.125-0.853)$ & 0.022 \\
\hline
\end{tabular}

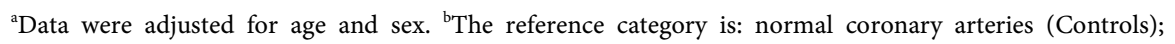
$\mathrm{P}$-Value is statistically significant $(<0.05)$.

Table 4. Rs11209026 G/A and dyslipidemia status in ACS patients and controls.

\begin{tabular}{ccccc}
\hline rs11209026 & Dyslipidemia (+) & Dyslipidemia $(-)$ & OR $(95 \% \mathrm{CI})^{\mathrm{a}}$ & $\mathrm{P}^{\mathrm{a}}$ \\
\hline $\begin{array}{c}\text { ACS patients } \\
\text { Genotypes }\end{array}$ & 96 & 64 & & \\
GG & $88(91.7)$ & $59(92.2)$ & 1 & \\
AG & $8(8.3)$ & $5(7.8)$ & $1.067(0.330-3.451)$ & 0.913 \\
Alleles & & & 1 & \\
G & $184(95.8)$ & $123(96.1)$ & & \\
A & $8(4.2)$ & $5(3.9)$ & $1.064(0.337-3.356)$ & 0.915 \\
Controls & 44 & 36 & & \\
Genotypes & & & & \\
GG & $37(84.1)$ & $26(72.2)$ & & \\
AG & $7(15.9)$ & $10(27.8)$ & $0.452(0.148-1.378)$ & 0.163 \\
Alleles & & & & \\
G & $81(92.0)$ & $62(86.1)$ & $0.501(0.177-1.415)$ & 0.501 \\
A & $7(8.0)$ & $10(13.9)$ & &
\end{tabular}

${ }^{\text {a}}$ Data were adjusted for age and sex. 
genotypes and alleles of the SNP between controls with dyslipidemia and those without dyslipidemia (Table 4). Based on the information contained in this data, rs11209026 G/A SNP is not involved in the development of dyslipidemia associated ACS.

\section{Discussion}

In the recent year, several researches have indicated that IL-23 can be involved in the development of atherosclerosis. For instance, patients with peripheral arterial disease have heightened serum levels of IL-23, and increased IL-23 expression is seen in human carotid lesions [11] [12]. In a mouse model of ischemic stroke, IL-23 levels were upregulated in both the circulation and in the brain [13]. While, effects of IL-23R on ACS have not been reported. The results of the present study revealed an association between rs11209026 G/A SNP of the IL-23 receptor gene and individual susceptibility to ACS. Our results were obtained by analyzing 160 ACS participants (included: 80 UAP patients and $80 \mathrm{MI}$ patients) and 80 healthy controls, and showed that rs11209026 G/A SNP in the IL-23R was associated as a protective factor for ACS. To further characterize the effect of rs11209026 G/A polymorphism on dyslipidemia-associated ACS, we found no correlated between rs11209026 G/A SNP and dyslipidemia-associated ACS.

Furthermore, several studies have reported the association between IL-23R rs11209026 polymorphism and effect of atherosclerosis. Kave et al. have reported that the genetic variant of rs11209026 polymorphism may in fact cause protection against atherosclerosis progression in Iran population [10]. However, another study indicated that it was failed to reveal an association between MI and the rs11209026 G/A SNP in white British population [14]. While, our results showed that percentages of IL-23R rs11209026 GG and AG genotypes were significantly different between the ACS group and the control group ( $p<0.05)$, and the A allele frequency of the acute coronary syndrome group was lower than the control group $(4.1 \%$ vs. $10.6 \%, p=0.005)$, suggesting that polymorphism changes at this site may be associated with the hereditary susceptibility of acute coronary syndrome (Table 2). Interestingly, after adjusted the gender and age of these data by unconditional logistic regression, our data revealed that the rs11209026 AG genotype was a protective factor for acute coronary syndrome and subtype of ACS (Table 3). In addition, after analysis the differences in results between current study and other studies indicates that the genetic susceptibility of rs11209026 G/A polymorphism to atherosclerosis is not only related to the disease phenotype of selected patient's, but also related to the selected control group and the source of the study population.

Although rs11209026 G/A SNP may have functional and phenotypic consequences on ACS, the molecular basis of the genetic variation is remains unclear. The SNP rs11209026 G/A, located in exon 9 of the IL-23R, has been associated with strong protection against Crohn's disease (allele A), which means that allele $A$ is underrepresented in the disease population [15] [16]. We and others have recently observed protective nature of rs11209026 allele A. It is important to de- 
termine whether this allele itself has a protective function or merely a protective association. Furthermore, the A allele causes an amino acid change close to the end of the coding sequence of exon 9, which induce the more common (allele G) arginine at residue 381 becomes a glutamine (R381Q) [17] [18]. Then, Di Meglio et al. inspected healthy participants and compared IL-23 responsiveness in allele A carriers compared with allele $G$ carriers. Moreover, their results illustrated that reduced IL-17A and STAT3 phosphorylation in the presence of allele A and suggested that the protective effects of the IL-23R R381Q were mediated through selective attenuation of IL-23-induced Th17 effector function [19]. Choosing a similar population of healthy participants, Pidasheva et al. demonstrated that IL23R-Q381 (allele A) is a loss-of-function allele and furthermore revealed that carriers of A allele had decreased surface IL-23R expression while reserving similar levels of IL23RmRNA [20]. In addition, Raymond et al. explored this protective allele A was associated with mRNA splicing, and they illustrated that the presence of the A allele increases expression of the soluble form of IL23R mRNA (which then functions as a decoy receptor) and lowers the ability to develop a Th17 phenotype upon IL-23 stimulation [17]. Moreover, the present research revealed that the frequency of rs 11209026 allele A was lower in the acute coronary syndrome group and its subgroup than in the control group, and maybe was a protective factor for acute coronary syndrome. Therefore, we speculate that rs11209026 allele A plays a protective role in acute coronary syndrome through the molecular mechanism discussed above.

\section{Conclusion}

The current study indicated that rs11209026 G/A polymorphism was associated with the decreased risk of ACS and it seems that the high prevalence rate of variable allele A in the normal coronary arteries group (compared to ACS patients) created protection against atherosclerosis progression. However, the current study did not explore the relationship between the molecular mechanism of IL-23R gene polymorphism and the susceptibility to ACS, and the amount of specimens was small. Therefore, its association with the susceptibility to acute coronary syndrome and its molecular mechanism in pathological process of atherosclerosis need further verification.

\section{Funding}

This study was funded by the Medical and Health Science and Technology Plan Project of Jingzhou (No.JZCH2017-10).

\section{Conflicts of Interest}

The authors declare no conflicts of interest regarding the publication of this paper.

\section{References}

[1] Mozaffarian, D., Benjamin, E.J., et al. (2016) Executive Summary: Heart Disease and Stroke Statistics-2016 Update: A Report from the American Heart Association. 
Circulation, 133, 447-454. https://doi.org/10.1161/CIR.0000000000000366

[2] Tabas, I. and Lichtman, A.H. (2017) Monocyte-Macrophages and T Cells in Atherosclerosis. Immunity, 47, 621-634. https://doi.org/10.1016/j.immuni.2017.09.008

[3] Crea, F. and Liuzzo, G. (2013) Pathogenesis of Acute Coronary Syndromes. Journal of the American College of Cardiology, 61, 1-11. https://doi.org/10.1016/j.jacc.2012.07.064

[4] Su, S.A., Ma, H., Shen, L., et al. (2013) Interleukin-17 and Acute Coronary Syndrome. Journal of Zhejiang University Science B, 14, 664-669. https://doi.org/10.1631/jzus.BQICC701

[5] Behzadi, P., Behzadi, E. and Ranjbar, R. (2016) IL-12 Family Cytokines: General Characteristics, Pathogenic Microorganisms, Receptors, and Signalling Pathways. Acta Microbiologica et Immunologica Hungarica, 63, 1-25. https://doi.org/10.1556/030.63.2016.1.1

[6] Fragoulis, G.E., Siebert, S. and McInnes, I.B. (2016) Therapeutic Targeting of IL-17 and IL-23 Cytokines in Immune-Mediated Diseases. Annual Review of Medicine, 67, 337-353.

[7] Gaffen, S.L., Jain, R., Garg, A.V., et al. (2014) The IL-23-IL-17 Immune Axis: From Mechanisms to Therapeutic Testing. Nature Reviews Immunology, 14, 585-600. https://doi.org/10.1038/nri3707

[8] Abbas, A., Gregersen, I., Holm, S., et al. (2015) Interleukin 23 Levels Are Increased in Carotid Atherosclerosis: Possible Role for the Interleukin 23/Interleukin 17 Axis. Stroke, 46, 793-799. https://doi.org/10.1161/STROKEAHA.114.006516

[9] Zhang, M., Cai, Z.R., Zhang, B., et al. (2014) Functional Polymorphisms in Interleukin-23 Receptor and Susceptibility to Coronary Artery Disease. DNA and Cell Biology, 33, 891-897. https://doi.org/10.1089/dna.2014.2573

[10] Kave, M., Shadman, M., Alizadeh, A., et al. (2015) Analysis of the Association between IL-23R rs11209026 Polymorphism and Incidence of Atherosclerosis. International Journal of Immunogenetics, 42, 341-345. https://doi.org/10.1111/iji.12223

[11] Erbel, C., Dengler, T.J., Wangler, S., et al. (2011) Expression of IL-17A in Human Atherosclerotic Lesions Is Associated with Increased Inflammation and Plaque Vulnerability. Basic Research in Cardiology, 106, 125-134. https://doi.org/10.1007/s00395-010-0135-y

[12] Fischer, K., Przepiera-Bedzak, H., Sawicki, M., et al. (2017) Serum Interleukin-23 in Polish Patients with Systemic Lupus Erythematosus: Association with Lupus Nephritis, Obesity, and Peripheral Vascular Disease. Mediators of Inflammation, 2017, Article ID: 9401432.

[13] Ma, S., Zhong, D., Chen, H., et al. (2013) The Immunomodulatory Effect of Bone Marrow Stromal Cells (BMSCs) on Interleukin (IL)-23/IL-17-Mediated Ischemic Stroke in Mice. Journal of Neuroimmunology, 257, 28-35. https://doi.org/10.1016/j.jneuroim.2013.01.007

[14] Mangino, M., Braund, P., Singh, R., et al. (2008) Association Analysis of IL-12B and IL-23R Polymorphisms in Myocardial Infarction. Journal of Molecular Medicine, 86, 99-103. https://doi.org/10.1007/s00109-007-0264-4

[15] Raad, M.A., Chams, N.H. and Sharara, A.I. (2016) New and Evolving Immunotherapy in Inflammatory Bowel Disease. Inflammatory Intestinal Diseases, 1, 85-95. https://doi.org/10.1159/000445986

[16] Can, G., Tezel, A., Gurkan, H., et al. (2016) Investigation of IL23R, JAK2, and STAT3 Gene Polymorphisms and Gene-Gene Interactions in Crohn's Disease and 
Ulcerative Colitis in a Turkish Population. The Turkish Journal of Gastroenterology: The Official Journal of Turkish Society of Gastroenterology, 27, 525-536. https://doi.org/10.5152/tjg.2016.16327

[17] Yu, R.Y., Brazaitis, J. and Gallagher, G. (2015) The Human IL-23 Receptor rs11209026 A Allele Promotes the Expression of a Soluble IL-23R-Encoding mRNA Species. Journal of Immunology, 194, 1062-1068. https://doi.org/10.4049/jimmunol.1401850

[18] Abdollahi, E., Tavasolian, F., Momtazi-Borojeni, A.A., et al. (2016) Protective Role of R381Q (rs11209026) Polymorphism in IL-23R Gene in Immune-Mediated Diseases: A Comprehensive Review. Journal of Immunotoxicology, 13, 286-300. https://doi.org/10.3109/1547691X.2015.1115448

[19] Di Meglio, P., Di Cesare, A., Laggner, U., et al. (2011) The IL23R R381Q Gene Variant Protects against Immune-Mediated Diseases by Impairing IL-23-Induced Th17 Effector Response in Humans. PloS ONE, 6, e17160. https://doi.org/10.1371/journal.pone.0017160

[20] Pidasheva, S., Trifari, S., Phillips, A., et al. (2011) Functional Studies on the IBD Susceptibility Gene IL23R Implicate Reduced Receptor Function in the Protective Genetic Variant R381Q. PloS ONE, 6, e25038.

https://doi.org/10.1371/journal.pone.0025038 